\title{
Fracture precursor phenomena in marble specimens under uniaxial compression by means of Acoustic Emission data
}

\author{
Dimos Triantis \\ University of West Attica, Department of Electrical and Electronic Engineering, Laboratory of Electronic Devices and Materials, \\ 250 Thivon Avenue, Campus 2, 122 44, Athens, Greece \\ triantis@uniwa.gr, bttp://orcid.org/0000-0003-4219-8687 \\ Stavros K. Kourkoulis \\ National Technical University of Athens, School of Applied Mathematical and Physical Sciences, Department of Mechanics, \\ Laboratory for Testing and Materials, 5 Heroes of Polytechnion Avenue, Theocaris Bld., Zografou Campus, 15773 Athens, Greece \\ stakkour@central.ntua.gr, bttps://orcid.org/0000-0003-3246-9308
}

\begin{abstract}
Marble specimens are subjected to a specially designed stepwise loading protocol, in an attempt to detect fracture precursor phenomena taking advantage of Acoustic Emission (AE) data. The analysis is carried out in terms of the number of acoustic hits recorded and the time evolution of the improved b-value ( $b$-value), the cumulative energy of the acoustic signals and the F-function. During the stage of increasing load, intense acoustic activity is detected as the corresponding stress reaches the transitional phase from the linear to the nonlinear mechanical response of the material. When the stress is stabilized at levels exceeding $95 \%$ of the material's compressive strength, the acoustic activity is drastically reduced. During the first seconds of the stress stabilization stage the reduction follows an exponential law. Special attention is paid to the phases, where the occurrence of AE hits shows a strong increase. During these phases acoustic signals of low frequency and high RA are recorded, indicating that the micro-cracking process is of shear rather than of opening mode.
\end{abstract}

KeYwords. Acoustic Emission; Marble; Compression; I $b$-value; F-function; Micro-cracking classification.

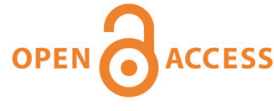

Citation: Triantis, D., Kourkoulis, S.K. Fracture precursor phenomena in marble specimens under uniaxial compression by means of Acoustic Emission data, Frattura ed Integrità Strutturale, 50 (2019) 537-547.

Received: 21.01.2019

Accepted: 18.05 .2019 Published: 01.10.2019

Copyright: (C) 2019 This is an open access article under the terms of the CC-BY 4.0, which permits unrestricted use, distribution, and reproduction in any medium, provided the original author and source are credited.

\section{INTRODUCTION}

$\mathrm{T}$ he mechanical response of brittle heterogeneous materials under compressive loading and the damage accumulation within their mass, which unavoidably accompanies the loading procedure, are of great interest in a wide range of engineering applications. Of particular interest are the quantitative description of the damage mechanisms activated and the early prediction of upcoming catastrophic failure. In this direction, series of damage assessment techniques have 
been long ago proposed and are being continually developed since then. Among them, the Acoustic Emission (AE) technique is perhaps the one most widely applied worldwide. Although the AE technique is introduced many decades ago, it is still developed and refined and today it is a flexible tool for monitoring and understanding the dynamic processes taking place within the mass of mechanically loaded structural elements. Moreover, the AE technique is considered as a unique tool providing warning signals about impending failure, especially for brittle materials [1-3]. Acoustic Emissions (AEs) are detected as series of short impulse energy packets, sequentially released when damage accumulation exceeds some critical limits leading to micro-cracking within the mass of materials subjected to mechanical loads. The detection of AEs is nowadays widely used for the spatiotemporal damage localization as well as for the assessment of the damage level (or in other words of the remaining load carrying capacity) in brittle materials such as concrete and rocks [4-7].

It is known that mechanical loading of rocks and rock-like materials generates a stress field, which is responsible for the activation of various micro-mechanisms, such as dislocation activity and formation of micro-cracks, which in turn are responsible for the release of elastic energy, traveling in the form of elastic waves and detected in the form of acoustic signals. The fracture process starts by the nucleation of micro-cracks, which grow and coalescence forming cracks leading to catastrophic fracture. Micro-cracking is the most important source of AEs at least for brittle materials, such as rocks [8]. Initially, tiny cracks are produced, which are gradually growing, reaching eventually a critical size, beyond which coalescence of the micro-cracks starts generating macro-cracks. In general, AEs are generated at different spatial and temporal scales.

It should be noted that the release of seismic waves related to an earthquake and the AEs detected in specimens of solids during their fracture process have remarkable similarities. The initial correlation of AEs and seismology was attempted by Ono and Ohtsu [9-11], who employed techniques used for earthquake data processing to elaborate laboratory AEs data. As a result, quite a few techniques applied in seismology from the civil engineering point of view are nowadays employed in the field of analysis of AEs data.

The number of incoming signals is a basic parameter when monitoring the acoustic activity. As the stress level increases approaching that causing failure of specimens or structural members, the number of AE hits recorded starts increasing at an increasing rate, depending on the material and the loading mode. Therefore, the rate of AE hits (dn/dt), can provide direct information on the frequency of micro-cracking activity in rocks. Graphical representation of dn/dt versus time provides information about the various stages or phases of micro-cracking activity in rocks. For further analysis of the failure mode, two additional AE parameters are widely used: the average frequency (AF) of the acoustic signals, defined as the number of counts divided by their duration, and the RA parameter (Rise Time/Amplitude) [12, 13]. It is accepted that cracks generating signals of relatively high AF and low RA are related to tensile cracking (Mode-I) while shear cracking (Mode-II) corresponds to lower AF and higher RA. Moreover, researchers in the field of Rock Mechanics use the so-called improved b-value analysis to quantify damage and describe the respective fracture processes $[1,3,14]$.

In this work, rock samples (marble) are subjected to uniaxial compression until fracture. The analysis of the AEs data focuses on how the acoustic activity evolves, especially in phases near the catastrophic fracture of the specimens. For this purpose, a special loading protocol of four successive steps is implemented, as it is described in next section. For the representation and analysis of the acoustic activity, besides the above mentioned parameters, a recently proposed F-function is used [7], which reflects the mean occurrence of AE hits, in a predefined time window of $\mathrm{N}$ consecutive hits.

\section{THE MATERIAL AND THE EXPERIMENTAL PROTOCOL}

$\mathrm{P}$ rismatic specimens made of Dionysos marble, of dimensions $40 \times 40 \times 100 \mathrm{~mm}^{3}$ were used. Dionysos marble is exclusively used for the restoration of the Athenian Acropolis monuments. Its composition is $98 \%$ calcite, $0.5 \%$ muscovite, $0.3 \%$ sericite, $0.2 \%$ quartz and $0.1 \%$ chlorite. Detailed characteristics of the material are given by Exadaktylos et al. [15]. During a preliminary experimental protocol with standardized specimens, the strength of the specific material's batch under monotonic uniaxial compression was determined, and it was found varying between $62.5 \mathrm{MPa}$ and 65.1 $\mathrm{MPa}$, with an average of about $63.8 \mathrm{MPa}$.

An Instron DX 300 loading frame was used for the main experimental protocol. The strain developed was measured using Kyowa strain gauges attached on the Microlink-770,120 $\Omega$ resistor bridge. An acoustic sensor was attached in the central cross section of the specimens with the aid of vacuum grease. Preamplifier with $40 \mathrm{~dB}$ gain and analogue band-pass filters in the $20-400 \mathrm{kHz}$ range was also used. The AE equipment and software were by Mistras Group, Inc.

The specimens were subjected to uniaxial compression until fracture, following a specially designed protocol of four discrete consecutive stages [16]: During the first stage (A), the load was applied at a constant rate, resulting to a stress rate of $0.44 \mathrm{MPa} / \mathrm{s}$, up to a stress level of $60.5 \mathrm{MPa}$. During the second stage (B), the duration of which was equal to about $120 \mathrm{~s}$, the stress was kept constant at $60.5 \mathrm{MPa}$. At the end of stage $\mathrm{B}$ the stress level was increased slightly at $63.5 \mathrm{MPa}$ of 
(stage C), with the same rate with that used in stage A $(0.44 \mathrm{MPa} / \mathrm{s})$. Finally, during the last stage (D) the stress level was again kept constant at $63.5 \mathrm{MPa}$, until the fracture of the specimens.

The time evolution of the stress during all four stages, for a characteristic specimen, is depicted in Fig.1, in juxtaposition to the respective evolution of the axial strain (as it was recorded by the strain gauge) for the whole loading procedure until the fracture of the specimen. The respective stress-strain curve is shown in Fig.2. It is noted that for the major portion of stage A the material's response is perfectly linear (excluding the unavoidable non-linearity accompanying the very early loading steps during uniaxial compression) until the stress reaches the level of $57 \mathrm{MPa}$, corresponding to about $90 \%$ of the fracture stress (point $\mathrm{K}$ in Fig.1)). The modulus of elasticity determined from this linear region of the stress-axial strain curve is equal to about $74 \mathrm{GPa}$ (Fig.2), in good agreement with previous experimental protocols by Kourkoulis et al. [17].

In stage $\mathrm{B}$, i.e., under constant stress of $60.5 \mathrm{MPa}$, the axial strain appears almost constant. However, thorough study of the respective data indicates that the strain during stage $\mathrm{B}$ is actually increasing (although imperceptibly), though at a gradually decreasing rate. In stage $\mathrm{D}$, i.e., under constant stress of $63.5 \mathrm{MPa}$, the strain is increasing, at rates much higher compared to those of stage $\mathrm{B}$. The time variation of the axial strain rate during stages $\mathrm{B}$ and $\mathrm{D}$, namely the stages during which the stress is kept constant, is plotted in Fig.3. It is very interesting to emphasize the significant increase of the axial strain rate during the last steps of stage D, which eventually leads to the fracture of the specimen under constant stress (!), about 60 seconds after the stabilization of the stress level (or, equivalently, 25 seconds before the fracture of the specimen).

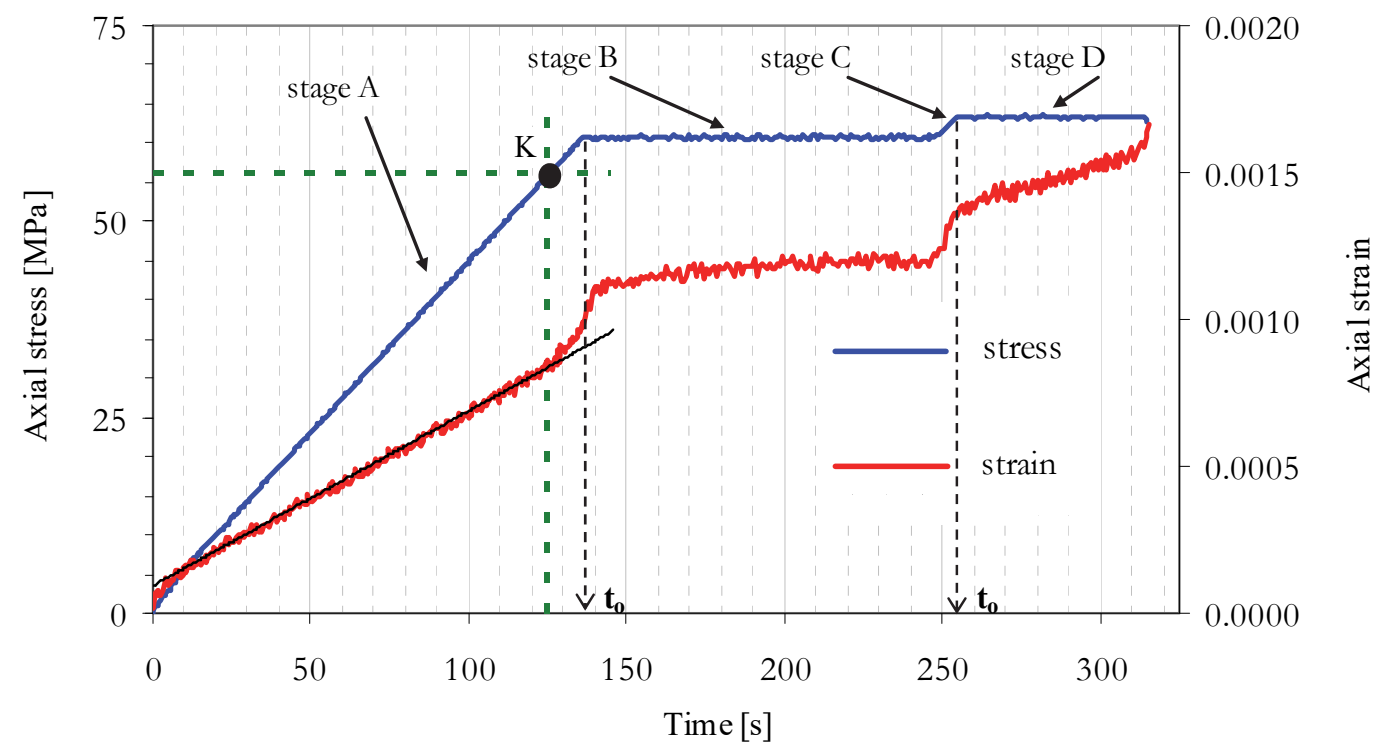

Figure 1: Stress and strain versus time. The four loading stages of the loading scheme are clearly visible.

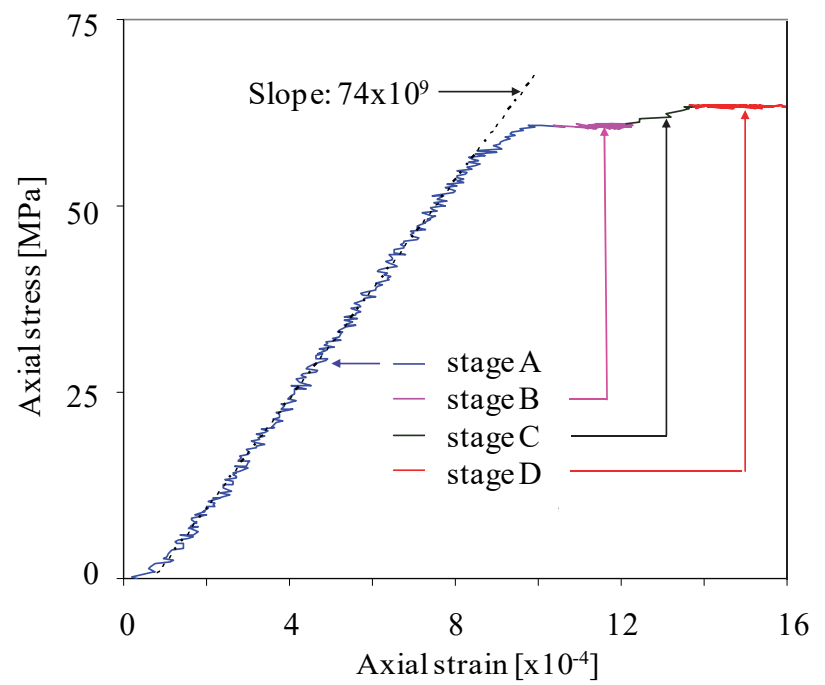

Figure 2: Axial stress versus axial strain for a typical experiment. The colour code is used to distinguish the four stages of the loading protocol exhibited in Fig.1. 


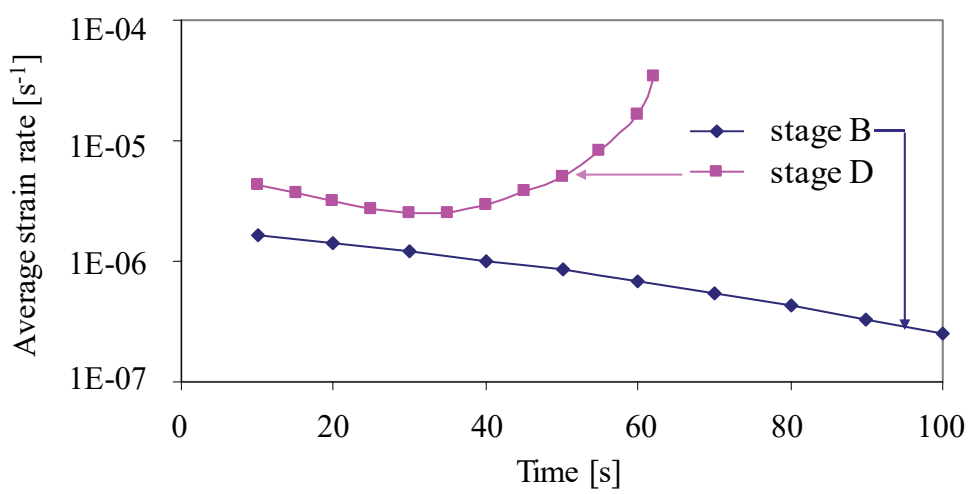

Figure 3: Time evolution of the average strain growth rate $\langle\Delta \varepsilon / \Delta \mathrm{t}\rangle$, in stages $\mathrm{B}$ and $\mathrm{D}$.

\section{EXPERIMENTAL RESULTS AND DISCUSSION}

\section{Analysis in terms of the Ib-value and the cumulative energy of the acoustic signals}

he number of AE hits recorded during uniaxial compression tests on rocks is usually large with inconsistent shapes of the respective waveforms and with parameters varying within wide limits. In the present study AE hits with duration less than $10 \mu \mathrm{s}$ and count number less than 2 were ignored [18], in order to exclude from the analysis $\mathrm{AE}$ hits that would probably be of parasitic nature. The number of $\mathrm{AE}$ hits, of amplitude equal or higher than $40 \mathrm{~dB}$ that were finally used after the above filtering, was equal to about 3500. For the specific experiment discussed in previous section, this number was equal to 3335. The allocation of these hits to each one of the four stages of the loading protocol is shown in Table 1, together with the average amplitude, the average energy and average duration of the hits of each stage.

\begin{tabular}{ccccc}
\hline Stage & AE hits number & Average amplitude $(\mathrm{dB})$ & Average energy (aJ) & Average duration $(\mu \mathrm{s})$ \\
A & 893 & 49.3 & 3098 & 4734 \\
B & 730 & 48.0 & 621 & 1966 \\
C & 174 & 50.9 & 7027 & 11112 \\
D & 1538 & 49.7 & 25095 & 5645 \\
\hline
\end{tabular}

Table 1: Statistical data for the AE parameters.

To further explore the details of the acoustic activity, an improved b-value (denoted from here on as $\mathrm{I} b$-value) analysis is carried out, as it was already discussed for the specific protocol by Triantis [16]. In this direction, the total number of AE hits was divided into groups of sequential hits. Each group included 100 hits and the sliding step was set equal to 1 . This means that the first group (providing the first value of $\mathrm{I} b$ ) includes the first 100 hits (i.e., the hits with order from $\mathrm{i}=1$ to $i=100$ ), the second group (providing the second value of $\mathrm{I} b$ ) includes the hundred hits with order from $i=2$ to $i=101$, etc. The $\mathrm{I} b$-values were then calculated using the familiar equation:

$$
I b=\frac{\log N\left(\mu-\alpha_{1} \sigma\right)-\log N\left(\mu+\alpha_{2} \sigma\right)}{\left(\alpha_{1}+\alpha_{2}\right) \sigma}
$$

In Eq.(1) $\mu$ is the mean amplitude, $\sigma$ is the standard deviation and $\alpha_{1}, \alpha_{2}$ are constants the numerical value of which is usually set equal to 1 [1]. Then, each $\mathrm{I} b$-value was paired to a time instant corresponding to the mean value of the time instants of occurrence of the hundred sequential hits of each group.

The results of the above procedure are shown in Fig.4, in which the time evolution of the I $b$-value is plotted for all four stages of the loading protocol, in juxtaposition to the respective evolution of the cumulative energy of the acoustic signals. The abrupt reductions of the $\mathrm{I} b$-values, towards levels approaching the critical limit of 1, observed in Fig.4, are always accompanied by strong increase of the cumulative energy of the acoustic signals. This is observed as one approaches the end of stage A (when the stress exceeds about $90 \%$ of the fracture stress), during stage C and, finally, at the end of stage D during 


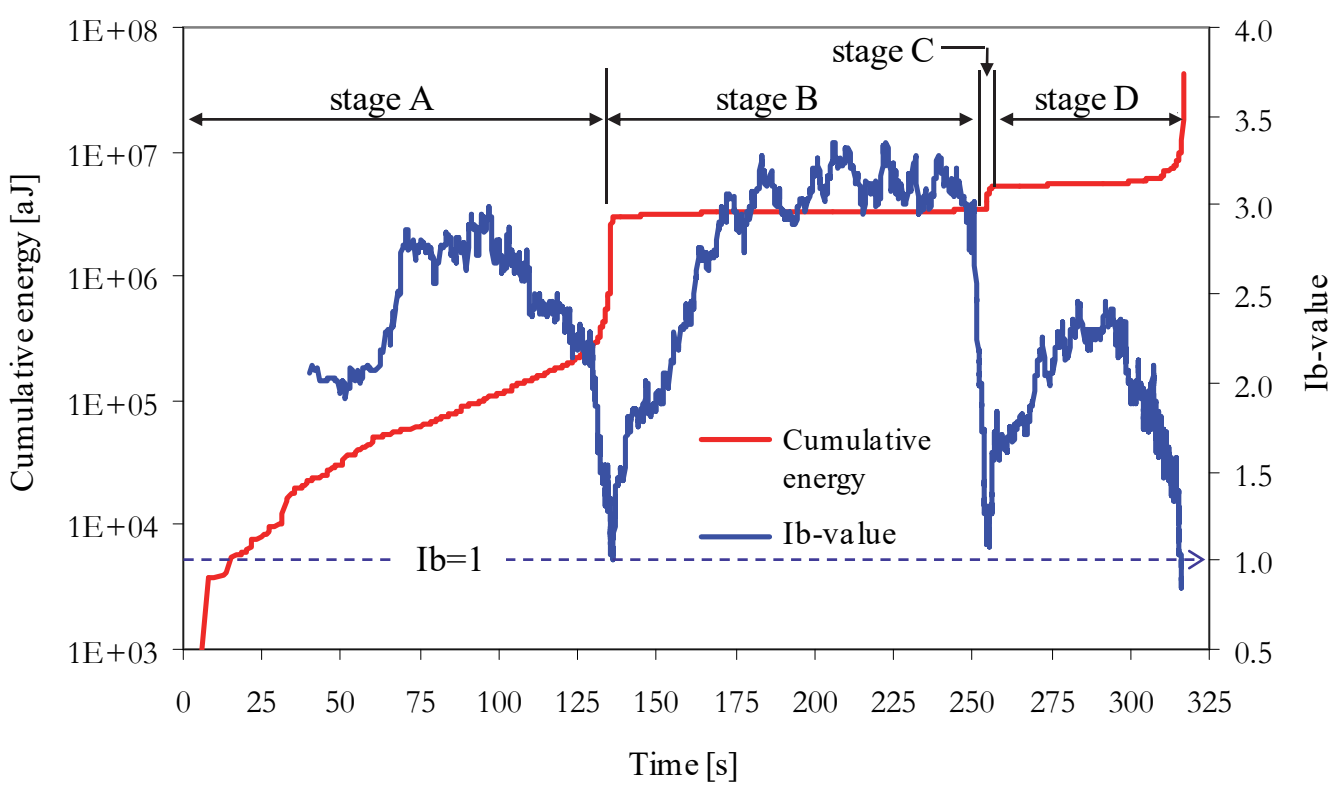

Figure 4: Time evolution of the cumulative energy and the $\mathrm{I} b$-values during the four stages of the loading scheme.

during the very last seconds before the macroscopic fracture of the specimen. The as above behaviour could be considered as corresponding to a transitional phase, from the stage of cracks formation to that of the final fracture of the specimen. At the end of this transitional phase preliminary crack-coalescence is possible.

Special attention is next paid to the time evolution of the cumulative energy (CE) of the acoustic signals in stages B and D, i.e., the stages of constant stress level. For both stages, it appears that the time evolution of the specific parameter is governed by a power law of the following form:

$$
C E=L\left(t-t_{o}\right)^{m}
$$

In Eq.(2) $\mathrm{L}$ and $\mathrm{m}$ are constants while $\mathrm{t}_{\mathrm{o}}$ corresponds to the time instant of onset of each constant stress stage (see Fig.1). Plotting Eq.(2) on log-log scales (Fig.5a) indicates that the time evolution of the cumulative energy (CE) of the acoustic signals during the constant stress stage B consists, in fact, of two distinct branches of different values of the exponent m. The first branch is of relatively limited duration $\left(\mathrm{t}-\mathrm{t}_{\mathrm{o}}<1 \mathrm{~s}\right)$ and corresponds to an exponent value $\mathrm{m}=0.70$. The second branch $\left(\mathrm{t}-\mathrm{t}_{\mathrm{o}}>1 \mathrm{~s}\right)$, corresponds to an exponent value $\mathrm{m}=0.31$. Attention is drawn to the fact that the starting point for calculating and drawing the time evolution of the cumulative energy is the time instant $t_{0}$ (the beginning of stage B), while the starting point of the horizontal axis $\left(t=t_{0}\right)$ corresponds, also, to the onset of stage $B$.

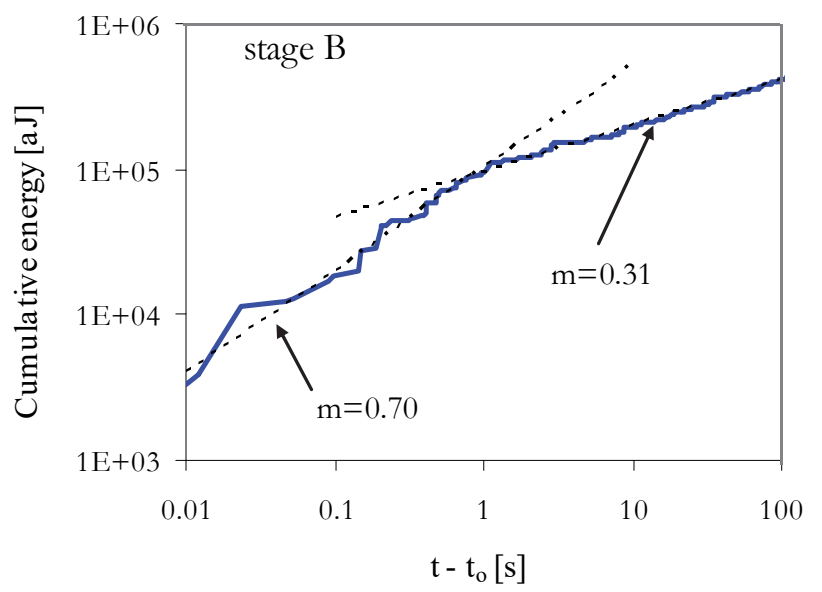

(a)

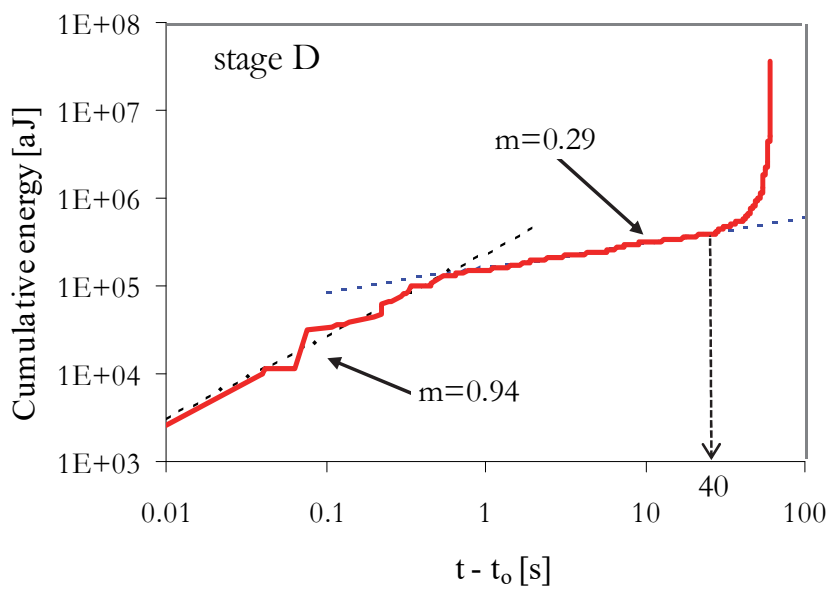

(b)

Figure 5: Time evolution of the cumulative energy in stages B (a) and D (b). 
A similar pattern prevails in stage $\mathrm{D}$ with exponent values $\mathrm{m}=0.94$ and $\mathrm{m}=0.29$, respectively (Fig.5b). Deviation from the law of Eq.(2) is only observed in this stage for $\mathrm{t}-\mathrm{t}_{\mathrm{o}}>40 \mathrm{~s}$ with the cumulative energy increasing quite rapidly. This deviation is obviously correlated to the fact that at this time instant the average rate of strain growth starts increasing rapidly (see Fig.3) indicating entrance of the system (specimen) into a state of impending failure, and providing thus a potentially interesting pre-failure indicator.

\section{Frequency of occurrence of AE hits - Analysis in terms of the F-function [7]}

In this section, a new way of exploring details of the acoustic activity is employed, based on the recently introduced Ffunction, which represents an average frequency of the $\mathrm{AE}$ hits, in a window of $\mathrm{N}$ successive hits [7]. To compute and plot the F-function the inter-event times of a sufficient number of $\mathrm{N}(\mathrm{N}>10)$ consecutive hits are used, in conjunction to the "sliding window" technique. Each value of the F-function is paired to an average time instant of the time instants of the $\mathrm{N}$ successive hits used to calculate the specific value of the F-function. Analytic description of how the F-function is determined can be found in refs. [7, 19]. This approach appears to be advantageous, since it allows for a continuous representation of the acoustic activity in a given time period rather than for a quantized presentation of the respective data, which characterizes the familiar plots of hits per second. This presentation is particularly useful when intense AE activity is recorded within a very short time interval. It offers, also, valuable information concerning the time evolution of the acoustic activity just before the failure of the samples, providing direct information on the frequency of micro-cracking activity within the specimen. Quite a few research teams worldwide are already using the F-function to study various aspects of the acoustic activity, especially in brittle materials [20-23].

The way according to which the F-function varies throughout the experiment discussed in previous sections is shown in Fig.6. The first significant and abrupt increase of the value of the F-function is observed in stage A and, in particular, after the $125^{\text {th }}$ second (denoted from here on as $t^{*}$ ). This time instant corresponds to the transition phase from the linear to the non-linear response of the material (see point K, Fig.1). Moreover, at the beginning of stage C, the F-function increases, also, considerably. At the beginning of stages B and D (stages of constant stress level), the F-function exhibits a rapid decrease and re-establishes low values, due to the sparse occurrence of AE. In particular, in stage D, the F-function, after a short period with low values, shows a gradual increase attaining a maximum value just a few seconds prior to the macroscopic fracture of the specimen, providing another interesting pre-failure indicator. Due to its increased importance the behaviour of the F-function during the last seconds of stage $\mathrm{D}$ will be discussed thoroughly in next paragraphs.

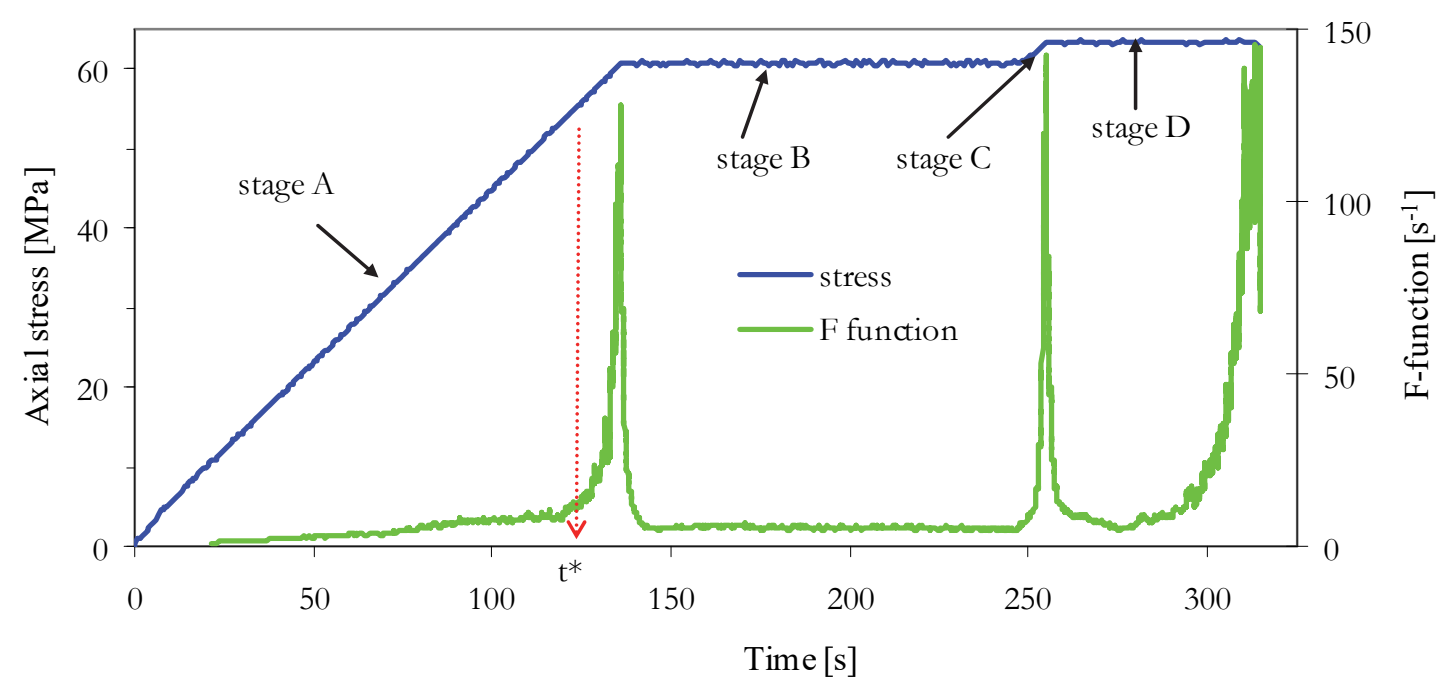

Figure 6: Time evolution of F-function during the experiment.

The time evolution of the F-function and the respective one of the $\mathrm{I} b$-values exhibit several qualitative similarities, as it is shown in Fig.7. More specifically, the sharp decrease of the $\mathrm{I} b$-values observed at the end of stages $\mathrm{A}$ and $\mathrm{D}$, as well as during stage $\mathrm{C}$, is combined with the corresponding intense increase of the acoustic activity expressed by the F-function. Furthermore, the intense decrease of the F-function at the beginning of stages $\mathrm{B}$ and $\mathrm{D}$ is combined with the gradual increase of the $\mathrm{I} b$-values. Special attention must be paid to the last seconds of stage $\mathrm{D}$, at which an abrupt decrease of the $\mathrm{I} b$-values, to levels approaching 1 , is observed. At the same time, as far as the F-function is concerned, a strong increase is recorded, leading to a local peak, which designates and presages the upcoming failure of the specimen [7]. 


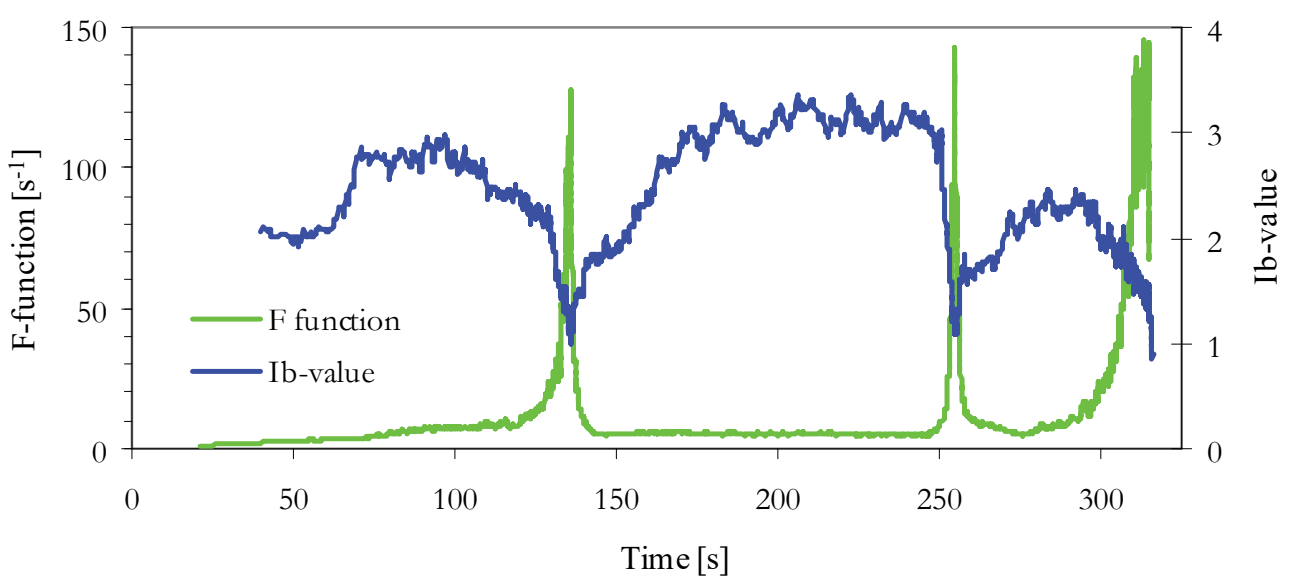

Figure 7: Time evolution of the F-function in juxtaposition to that of the $\mathrm{I} b$-value.

Considering now the systematic reduction exhibited by the F-function at stages B and D, further analysis of the experimental results was undertaken, indicating that during the first two (2) seconds after the stabilization of the stress level, the F-function decreases following a power law of the form:

$$
F(t)=A \exp \left(-\frac{t}{\tau}\right)
$$

The above law is plotted in Fig.8 for both stages B (Fig.8a) and D (Fig.8b) using logarithmic scale for the values of the Ffunction. In this figure, the origin of the time scale (i.e., $\mathrm{t}=0$ ) corresponds to the time instant $\mathrm{t}_{\mathrm{o}}$ (for each stage) at which the stress attains its maximum value and is then stabilized, practically corresponding to the time instant at which the maximum values of the F-function are attained. The values of the parameter $\mathrm{A}$ and the time constant $\tau$ of Eq.(3), as obtained by means of proper curve fitting, are equal to $A=128.7 \mathrm{~s}^{-1}, \tau=0.98 \mathrm{~s}$ for stage $\mathrm{B}$ and $\mathrm{A}=141.6 \mathrm{~s}^{-1}, \tau=1.23 \mathrm{~s}$, for stage $\mathrm{D}$. As it is expected, the highest value of the time constant $\tau$ is observed in stage $\mathrm{D}$ due to the considerably increased acoustic activity that is recorded in this stage (recall that, after a while, fracture of the specimen occurred).

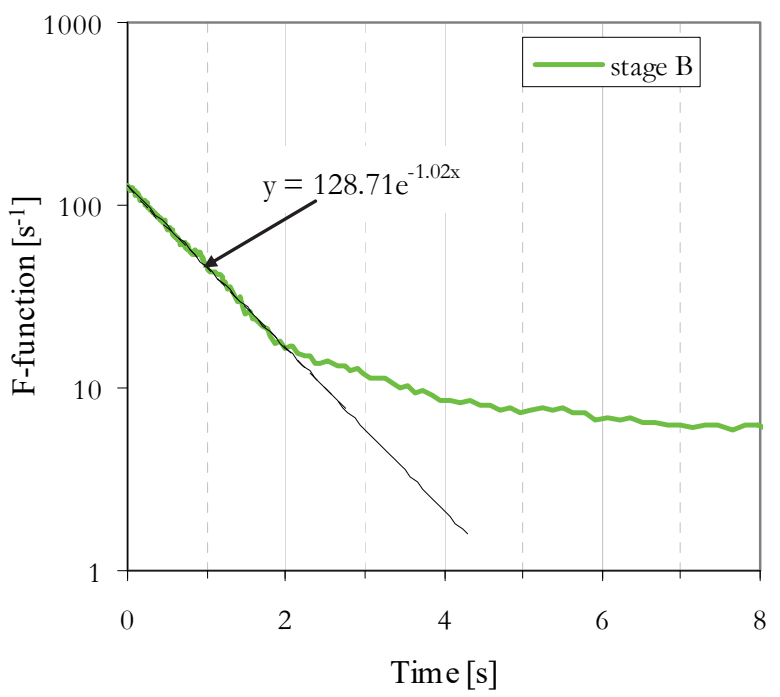

(a)

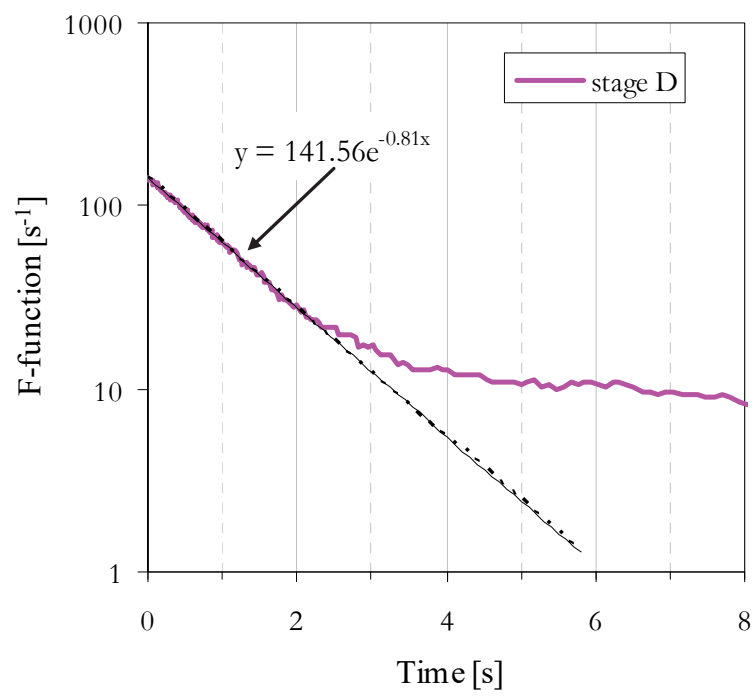

(b)

Figure 8: Restoration of the F-function during the first seconds following stress stabilization in stages B (a) and D (b).

As a last step, it is worth studying the time evolution of the F-function during the very last seconds prior to the failure of the specimen (i.e., the very last time interval of stage D). In a recent paper by Triantis and Kourkoulis [7], it was indicated that the F-function exhibits a peak just before the fracture, while before the occurrence of this peak there is a sharp increase of the F-function, which in terms of the time-to-failure parameter, $\mathrm{t}_{\mathrm{f}} \mathrm{t}$, is governed by a power law of the form: 


$$
F=c\left(t_{f}-t\right)^{-n}
$$

where $t_{f}$ is the time instant corresponding to the fracture of the sample and $t, n$ are numerically obtained constants. A similar response is observed in the present experimental protocol. This is clearly seen in Fig.9, in which the F-function is plotted versus the $\left(t_{-}-t\right)$ parameter on log-log scales. Plotting the F-function versus the specific parameter offers a "magnified" picture of what happens during the very last instants before macroscopic fracture. It is observed from Fig. 9 that from the critical time instant at which the F-function starts increasing (i.e., about $40 \mathrm{~s}$ before the specimen's macroscopic fracture) the law of Eq.(4) prevails, with an exponent value equal to $n=1.4$, close enough to the value of $n=1.2$ determined in the protocol described in ref. [7] for marble specimens under monotonic uniaxial compression. From this time instant on, i.e., for $\left(t_{\mathrm{f}}-\mathrm{t}\right)<5 \mathrm{~s}$, the F-function exhibits a stabilisation trend at relatively high values. Finally, during the very last tenths of a second before macroscopic fracture (i.e., $\left(\mathrm{t}_{\mathrm{f}}-\mathrm{t}\right)<0.7 \mathrm{~s}$ ) the values of the F-function are reduced, as a consequence of the high values of the respective inter-event times, due to the fact that long-duration acoustic hits are recorded (again in accordance with the behaviour observed in ref. [7]).

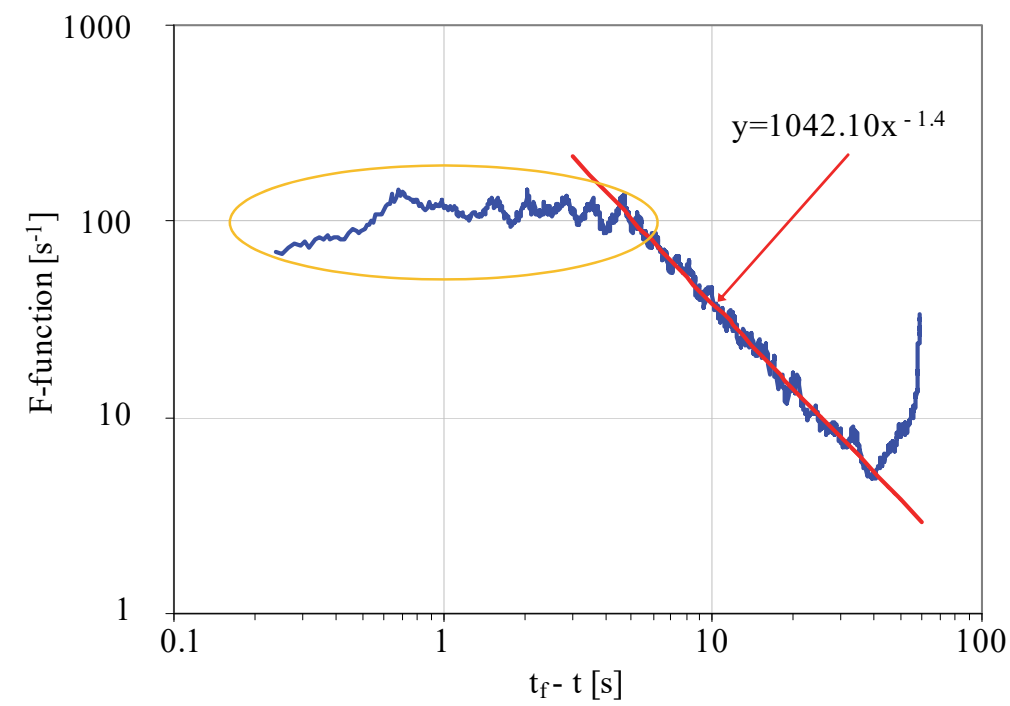

Figure 9: The F-function versus the $\left(t_{\mathrm{f}}-\mathrm{t}\right)$ parameter on a $\log -\log$ scale, during the last seconds before the sample failure (i.e., during the last time interval of stage D).

\section{Classification of the cracking modes by correlating Average Frequency and $\mathrm{R} A$ values to the values of the F-function}

The AE technique is already widely used to classify the cracking modes according to the RILEM TC 212-ACD Recommendation, with very satisfactory results, for example in concrete [24]. According to the specific recommendation, the classification of micro-cracking in Mode-I or Mode-II is achieved through the correlation of the parameter RA (Rise Time/Amplitude) of the acoustic signals to their Average Frequency (AF). In order to investigate how the average values of RA and $\mathrm{AF}$ are correlated in the various loading stages of the experimental protocol considered here, in which both high and low acoustic activity is observed, the following procedure is followed:

As a first step, the maximum value $\mathrm{F}_{\max }$ of the F-function is determined. For the specific experiment, considered in previous sections, the time instant at which the F-function was maximized was found equal to about $\mathrm{t}=319 \mathrm{~s}$ and the corresponding value of the F-function was $F_{\max }=145.7 \mathrm{~s}^{-1}$. For each one of the four stages of the loading protocol the acoustic hits were classified into three categories: The first one includes the hits that are recorded when the acoustic activity is low or, equivalently, the normalized function $\mathrm{f}=\mathrm{F} / \mathrm{F}_{\max }$ varies in the $0<\mathrm{f}<0.1$ range. The second category includes the hits recorded when $0.1 \leq \mathrm{f}<0.5$ (i.e., the acoustic activity is of moderate intensity), and the third one includes the hits recorded when $\mathrm{f} \geq 0.5$ (i.e., the acoustic activity is intense). As a second step, for each one of these three categories and for each stage of the loading protocol the average values of RA and AF are calculated. In Fig.10 the mean value of the AF is plotted against the respective mean value of the RA for all three categories and all four stages. A straight line of slope equal to $\mathrm{k}=0.5 \mathrm{kHz} \cdot \mathrm{mV} / \mu \mathrm{s}$ is plotted, starting from point $(0,0)$, in order to study the ratio between the RA and AF values and properly classify the cracking modes within the marble volume. It is noted here that the appropriate slope of this line is an open issue for research, as there is not a generally accepted rule. In general, it is believed that this slope is to be determined taking into account the type of the material and the type of the experiment [25]. 

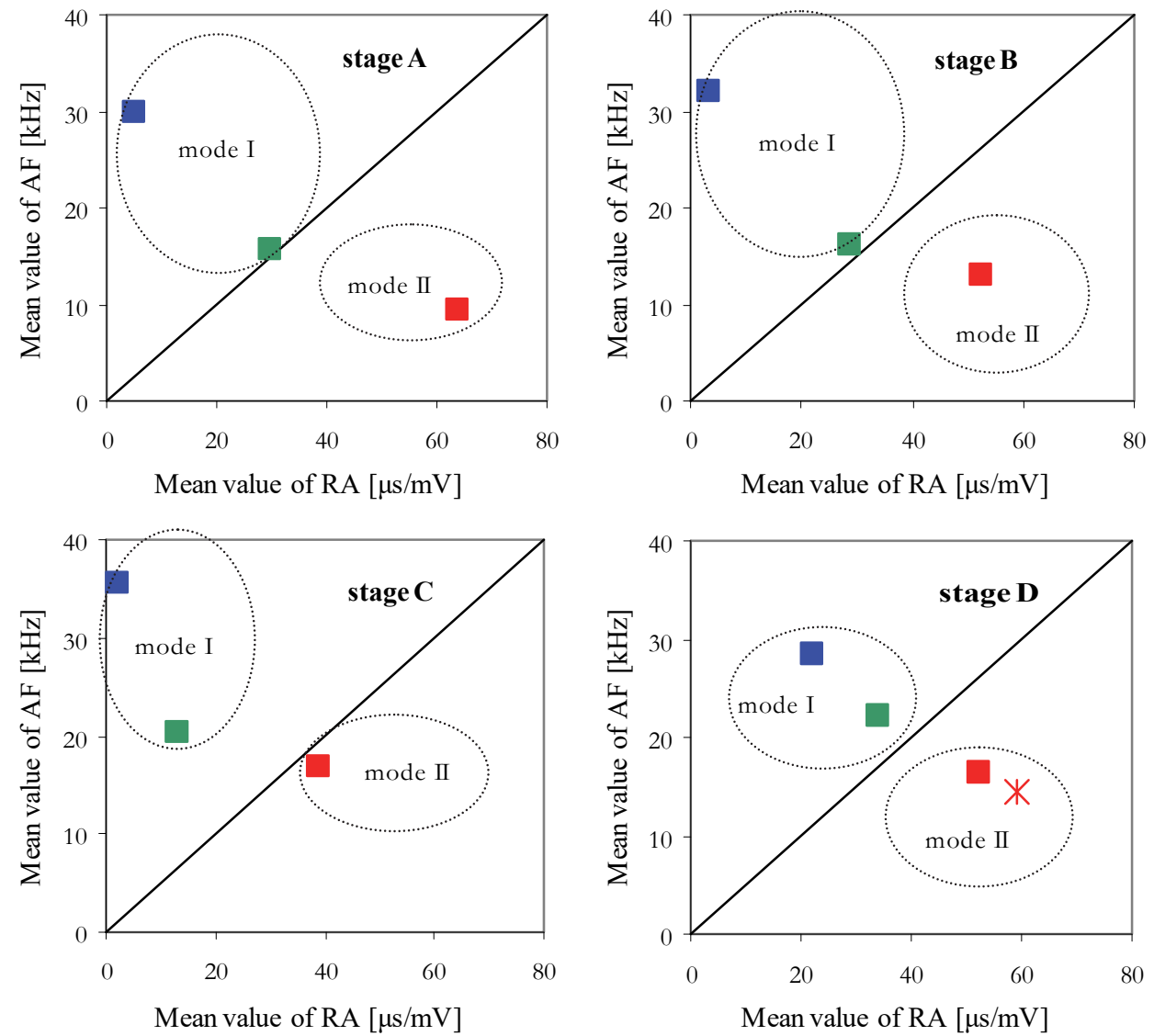

Figure 10: Classification of cracking modes according to the RILEM TC 212-ACD Recommendation (i.e., with the aid of the relation between the AF and RA parameters), in the four stages of the loading protocol ((a), (b), (c), (d) correspond to the stages A, B, C, D, respectively (see Fig.1)). The blue squares correspond to $f<0.1$, the green ones to $0.1 \leq f<0.5$ and the red ones to $f \geq 0.5$. The red star in Fig.10d (i.e., for stage D) corresponds to the average values of AF and RA during the last 5 seconds before the catastrophic fracture of the specimen (see Fig.9).

For $\mathrm{f} \geq 0.5$ in all four stages, the mean values of $\mathrm{RA}$ and $\mathrm{AF}$ correspond to gradients $\mathrm{k}<0.5 \mathrm{kHz} \cdot \mathrm{mV} / \mu \mathrm{s}$. This leads to the conclusion that, at least for the specific material, when the acoustic activity is intense, the shear type of cracking (Mode-II) is dominant. In contrast, when the acoustic activity is low $(f<0.1)$, it is clear that all acoustic signals are classified as ModeI ones. Particularly, during the last 5 seconds before the final fracture of the sample, the F-function shows a stabilization trend but with strong fluctuations $(\mathrm{F}=110 \pm 35) \mathrm{s}^{-1}$ (see Fig.9), and the shear type of micro-cracking (Mode-II) is prominent. The mean values of RA and AF at this time interval are depicted Fig.10d with the aid of a red star.

A more detailed description of the results of classification of cracking modes according to the characteristics of the AE hits in the four stages of the experiment discussed here is presented in Table 2. Again, it is seen from this table that when the acoustic activity is high ( $\mathrm{f}>0.5$ ) the Mode-II cracking is dominant (exceeding $50 \%$ ) for all four stages of the loading scheme. On the contrary, for $\mathrm{f}<0.5$ the majority of $\mathrm{AE}$ hits corresponds to Mode-I cracking. The shear mode exists also but it corresponds to a relatively low proportion ranging from about $6 \%$ to about $31 \%$.

\begin{tabular}{ccccc}
\hline \multirow{2}{*}{ Stage } & \multicolumn{2}{c}{$\mathrm{f}>0.5$} & \multicolumn{2}{c}{$\mathrm{f}<0.5$} \\
& Mode-I & Mode-II & Mode-I & Mode-II \\
A & $22 \%$ & $78 \%$ & $85 \%$ & $15 \%$ \\
B & $25 \%$ & $75 \%$ & $94 \%$ & $6 \%$ \\
C & $44 \%$ & $56 \%$ & $87 \%$ & $13 \%$ \\
D & $35 \%$ & $65 \%$ & $69 \%$ & $31 \%$ \\
\hline
\end{tabular}

Table 2: Classification of AE hits. 


\section{CONCLUSIONS}

he experimental protocol described here was designed in order to gather data regarding the response of rock-like materials under stepwise uniaxial compression, at load levels closely approaching those causing fracture. As a first step, the acoustic activity was analyzed in terms of the improved b-value ( $\mathrm{I} b$-value) and the cumulative energy of the acoustic signals. It was demonstrated that, when the acoustic activity is weak, the $\mathrm{I} b$-value varies in the $2<\mathrm{I} b<3$ interval. However, as the acoustic activity is intensified, the $\mathrm{I} b$-value drops rapidly, attaining values close to the critical value of 1 , i.e., to the limit designating the onset of formation of macro-cracks. As far as it concerns the cumulative energy of the acoustic signals, it was observed that during the stages of constant stress level, its time evolution is governed by a power law with two distinct branches. However, during the last seconds before fracture it exhibits an abrupt increasing trend, in spite of the fact that the stress is constant.

The detection of signals that could play the role of pre-failure indicators was also considered. This was attempted it terms of the time evolution of the I $b$-value, the cumulative energy of the acoustic hits and the F-function. It was definitely indicated that all three parameters just mentioned provide interesting and clear indices which could be considered as timely warning signals designating entrance of the system (specimen) into the stage of impending failure. Especially the F-function, well before macroscopic fracture is visible, exhibits an increasing trend which is governed by a power law. Then, a few seconds (or even tenths of a second) before the onset of macroscopically visible fracture, it reaches a maximum value and starts decreasing.

Concerning the stages at which the specimen is under constant externally applied stress, the mean frequency of the AE hits, as it is expressed by the F-function, exhibits an exponential decrease (during the first one or two seconds of the constant stress stage). Then, it shows a stabilization tendency at fairly low values. An exception is the stage at which the stress is stabilized at levels closely approaching its ultimate value (i.e., the compressive strength of the material). In this stage, after a time interval of about 40 seconds (almost $25 \mathrm{~s}$ before fracture), the F-function exhibits a sharp increase (under constant stress), thus presaging the final macroscopic collapse of the specimen.

Finally, the standardized approach for the classification of the damage process in terms of the RA-AF pair (which in fact determines the ratio of each type of damage, i.e., Mode-I or Mode-II micro-cracking), based on the RILEM TC 212-ACD recommendation, was applied. The classification showed that, for the phases with weak acoustic activity, Mode-I crackingh type appears as the dominant one, while, when the acoustic activity is intense, the majority of the acoustic signals corresponds to the Mode-II type of cracking.

\section{REFERENCES}

[1] Rao, M.V.M.S. and Lakschmi, P.K.J. (2005). Analysis of b-value and improved b-value of acoustic emissions accompanying rock fracture, Curr. Sci. India, 89, pp. 1577-1582.

[2] Colombo, I.S., Main, I. and Forde, M.C. (2003). Assessing damage of reinforced concrete beam using "b-value" analysis of Acoustic Emission signals, J. Mater. Civil. Eng., 15, pp. 280-286.

[3] Cox, S.J.D. and Meredith, P.G. (1993). Microcrack formation and material softening in rock measured by monitoring acoustic emissions, Int. J. Rock Mech. Min., 30, pp. 11-24.

[4] Aggelis, D.G., Mpalaskas A.C. and Matikas, T.E. (2013). Investigation of different fracture modes in cement-based materials by acoustic emission, Cement Concrete Res., 48, pp. 1-8.

[5] Stanchits, S., Dresen, G. and Vinciguerra, S. (2006). Ultrasonic velocities, acoustic emission characteristics and crack damage of basalt and granite, Pure Appl. Geophys., 163, pp. 975-994.

[6] Lockner, D. (1993). The role of acoustic emission in the study of rock fracture, Int. J. Rock Mech. Min., 30, pp. 883899.

[7] Triantis, D. and Kourkoulis, S. K. (2018). An alternative approach for representing the data provided by the acoustic emission technique, Rock Mech. Rock Eng., 51, pp. 2433-2438.

[8] Ono, K. (1979). Fundamentals of acoustic emission, In: Fundamentals of Acoustic Emission, Ono, K. (ed.), Los Angeles, USA, UCLA Publ.

[9] Ohtsu, M., Shigeishi, M., Iwase, H. and Koyanagi, W. (1991). Determination of crack location, type and orientation in concrete structures by acoustic emission, Mag. Concrete Res., 155, pp. 127-134.

[10] Ono, K. (1993). Trends of recent acoustic emission literature, J. Acoustic Emission, 12, pp. 177-198

[11] Ohtsu, M. (1994). New trends in non-destructive and in-place testing of concrete structures, In: Proc. Concrete Technology: New Trends, Industrial Applications, Aguado A., Gettu R. and Shah, S.P. (eds), London, E\&FN Spon. 
[12] Ohtsu, M. and Tomoda, Y. (2008). Phenomenological model of corrosion process in reinforced concrete identified by acoustic emission, ACI Mater. J., 105, pp. 194-199.

[13] Aggelis, D.G., Soulioti, D.V., Sapouridis, N., Barkoula, N.M., Paipetis, A.S. and Matikas, T.E. (2011). Acoustic emission characterization of the fracture process in fibre reinforced concrete, Constr. Build. Mater., 25, pp. 4126-4131.

[14] Main, I.G. (1989). A reinterpretation of the precursory seismic b-value anomaly from fracture mechanics, Geophys. J., 96, pp. 131-138.

[15] Exadaktylos, G.E., Vardoulakis, I. and Kourkoulis, S.K. (2001). Influence of nonlinearity and double elasticity on flexure of rock beams - II. Characterization of Dionysos marble, Int. J. Solids Struct. 38(22-23), pp. 4119-4145.

[16] Triantis, D. (2018). Acoustic emission monitoring of marble specimens under uniaxial compression. Precursor phenomena in the near-failure phase, Procedia Structural Integrity 10, pp. 11-17.

[17] Kourkoulis, S.K., Exadaktylos, G.E. and Vardoulakis, I. (1999). U-notched Dionysos-Pentelicon marble in three point bending: The effect of nonlinearity, anisotropy and microstructure., Int. J. Fracture, 98, pp. 369-392.

[18] Calabrese, L., Campanella, G. and Proverbio, E. (2012). Noise removal by cluster analysis after long time AE corrosion monitoring of steel reinforcement in concrete, Constr. Build. Mater., 34, pp. 362-371.

[19] Triantis, D., Stavrakas, I., Pasiou, E. D. and Kourkoulis, S.K. (2019). Assessing the acoustic activity in marble specimens under stepwise compressive loading, Mat. Design Process Comm., doi:https://doi.org/10.1002/mdp2.100.

[20] Zhang, J-Z., Zhou X-P., Zhou, L-S. and Berto, F. (2019). Progressive failure of brittle rocks with non-isometric flaws: Insights from acousto- optic-mechanical (AOM) data, Fatigue Fract. Eng. Mater. Struct., 42, pp. 1787-1802.

[21] Zhou, X-P., Zhang, J-Z., Qian, Q-H. and Niu, Y. (2019). Experimental investigation of progressive cracking processes in granite under uniaxial loading using digital imaging and AE techniques, J. Struct. Geol., 126, pp. 129-145.

[22] Wang, X., Wang, E., Liu, X. (2019). Damage characterization of concrete under multi-step loading by integrated ultrasonic and acoustic emission techniques, Constr. Build. Mater., 221, pp. 678-690.

[23] Niu Y., Zhou X-P., Zhou L-S. (2019). Fracture damage prediction in fissured red sandstone under uniaxial compression: acoustic emission b-value analysis, Fatigue Fract. Eng. Mater. Struct., DOI: 10.1111/ffe.13113.

[24] Ohtsu, M. (Chairman) (2010). Recommendations of RILEM Technical Committee 212-ACD: Acoustic emission and related NDE techniques for crack detection and damage evaluation in concrete: 3 . Test method for classification of active cracks in concrete structures by acoustic emission, Mater Struct. 43, pp.1187-1189.

[25] Aggelis, D.G. (2011). Classification of cracking mode in concrete by acoustic emission parameters. Mech. Res. Commun., 38, pp. 53-157. 